\title{
Documentación básica. \\ Razones médicas aceptables para el uso de sucedáneos de la leche materna
}

Organización Mundial de la Salud (OMS, 2009)

Fecha de publicación en Internet: 12 de noviembre de 2010

\section{Prefacio}

La Organización Mundial de la Salud (OMS) y el Fondo Internacional de las Naciones Unidas para Emergencias de la Infancia (UNICEF) desarrollaron una lista de razones médicas aceptables para el uso de sucedáneos de la leche materna, originalmente como anexo al paquete de herramientas para la Iniciativa Hospital Amigo del Niño (IHAN) en 1992.

La OMS y UNICEF acordaron actualizar la lista de dichas razones médicas por considerar que desde 1992 empezó a disponerse de nuevas pruebas científicas y que, además, se estaba actualizando el paquete de la IHAN. El proceso estuvo bajo el liderazgo de los departamentos de Salud y Desarrollo del Niño y del Adolescente (CAH) y de Nutrición para la Salud y el Desarrollo (NHD). En 2005 se compartió el borrador de una lista actualizada con los revisores de los materiales de la IHAN. En septiembre de 2007 la OMS invitó a un grupo de expertos de una variedad de campos y de todas las regiones de la OMS a participar en una red virtual de revisión de la lista. El borrador de la lista fue compartido con todos los expertos que aceptaron participar. Se prepararon varios borradores a partir de tres procesos relacionados: a) varias rondas de comentarios de los expertos; b) una compilación de revisiones técnicas y guías de orientación actualizadas de la OMS (véase la lista de referencias), y c) comentarios de otros departamentos de la OMS (Reducción de los Riesgos del Embarazo, Salud Mental, Medicamentos Esenciales), en general y en temas específicos o a raíz de preguntas especificas de los expertos.

La OMS no disponía de revisiones técnicas o guías orientadoras en algunos temas. En estos casos, se identificaron

Extracto autorizado del original "Razones médicas aceptables para el uso de sucedáneos de la leche materna", Organización Mundial de la Salud, 2009. El documento original completo puede obtenerse en http://whqlibdoc.who.int/hq/2009/who_fch_cah_09.01_spa.pdf y en http://www.who.int/nutrition/publications/infantfeeding/WHO_NMH_NHD_09.01_spa.pdf 
pruebas científicas consultando con los departamentos correspondientes de la OMS o con expertos externos en el área específica. Se utilizaron en particular las siguientes fuentes de pruebas científicas:

- La Base de Datos sobre Medicamentos y Lactancia (LactMed), un sitio Web de la Biblioteca Médica Nacional de los Estados Unidos de América, que es revisada por homólogos y contiene referencias completas sobre medicamentos a los que las madres lactantes podrían estar expuestas.

- Las Guías Clínicas Nacionales para el manejo de fármacos durante el embarazo, el parto y la lactancia, revisadas por el Departamento de Salud de Nueva Gales del Sur, Australia, 2006.

La lista final resultante fue compartida con revisores externos e internos para llegar a acuerdos, y es la que presentamos en este documento.

La lista de razones médicas aceptables para uso temporal o a largo plazo de sucedáneos de la leche materna está disponible tanto como una herramienta independiente para los profesionales de la salud que trabajan con madres y recién nacidos, como parte del paquete de la IHAN. Se espera actualizar esta lista hacia 2012.
Agradecimientos

Esta lista fue desarrollada por los departamentos de Salud y Desarrollo del Niño y del Adolescente y de Nutrición para la Salud y el Desarrollo de la OMS, en colaboración estrecha con UNICEF y los departamentos de Reducción de los Riesgos del Embarazo, de Medicamentos Esenciales y de Salud Mental y Abuso de Sustancias de la OMS. Contribuyeron a la elaboración de la lista actualizada los siguientes expertos: Philip Anderson, Colin Binns, Riccardo Davanzo, Carol Kolar, Ruth Lawrence, Lida Lhotska, Audrey Naylor, Jairo Osorno, Marina Rea, Felicity Savage, María Asunción Silvestre, Tereza Toma, Fernando Vallone, Nancy Wight, Anthony Williams y Elizabeta Zisovska. Todos ellos completaron la declaración de interés y ninguno identificó conflicto de interés.

\section{Introducción}

Casi todas las madres pueden amamantar con éxito, lo que significa iniciar la lactancia materna durante la primera hora de vida, dar lactancia materna exclusiva durante los primeros seis meses, y continuar con la lactancia (además de alimentación complementaria apropiada) hasta los dos años de edad o más. La lactancia materna exclusiva durante los primeros seis meses de vida es particularmente beneficiosa para madres y lactantes. 
Los efectos positivos de la lactancia materna en la salud de los niños y las madres han sido observados en todo lugar. La lactancia materna reduce el riesgo de infecciones tales como diarrea, neumonía, otitis, Haemophilus influenzae, meningitis e infección urinaria ${ }^{1}$. Protege también contra enfermedades crónicas futuras tales como diabetes mellitus tipo I, colitis ulcerosa y enfermedad de Crohn. La lactancia materna durante la infancia se asocia con una presión arterial media y un colesterol sérico total menores, y con menor prevalencia de diabetes mellitus tipo 2, sobrepeso y obesidad durante la adolescencia y la edad adulta ${ }^{2}$. La lactancia materna retrasa el retorno de la fertilidad de la mujer y reduce el riesgo de hemorragia postparto, cáncer de mama premenopáusico y cáncer de ovario ${ }^{3}$.

Sin embargo, un número pequeño de estados de salud del recién nacido y de la madre podría justificar que se recomendara no amamantar de manera temporal o permanente ${ }^{4}$. Estos casos, que afectan a muy pocas madres y sus bebés, se mencionan a continuación junto con otras enfermedades maternas que, aunque serias, no son razones médicas para el uso de sucedáneos de la leche materna.

Cuando se considere la interrupción de la lactancia, habrá que sopesar los riesgos de cualquiera de las enfermedades enumeradas a continuación en función de los beneficios que reportaría la lactancia materna.

\section{Afecciones infantiles}

1. Lactantes que no deben recibir leche materna ni otra leche excepto fórmula especializada:

- Lactantes con galactosemia clásica: se necesita una fórmula especial libre de galactosa.

- Lactantes con la enfermedad de la orina con olor a jarabe de arce: se necesita una fórmula especial libre de leucina, isoleucina y valina.

- Lactantes con fenilcetonuria: se requiere una fórmula especial libre de fenilalanina (se permite algo de lactancia materna, con monitorización cuidadosa).

2. Recién nacidos para quienes la leche materna es la mejor opción de alimentación, pero que pueden necesitar otros alimentos durante un periodo limitado, además de leche materna:

- Lactantes nacidos con peso menor de $1500 \mathrm{~g}$ (muy bajo peso al nacer).

- Lactantes nacidos con menos de 32 semanas de gestación (muy prematuros).

- Recién nacidos con riesgo de hipoglucemia debido a una alteración en la adaptación metabólica o un 
incremento de la demanda de glucosa, como los prematuros, los pequeños para la edad gestacional o los recién nacidos que han experimentado hipoxia significativa intraparto, así como aquellos que están enfermos o los hijos de madre diabética ${ }^{5}$, si la glucemia no se controla con lactancia materna o con alimentación con leche materna extraída.

\section{Afecciones maternas}

Las madres afectadas por alguna de las enfermedades mencionadas más abajo deberían recibir tratamiento según las recomendaciones habituales.

1. Afecciones maternas que podrían justificar que se evite la lactancia de forma permanente:

- Infección por VIHa: si la alimentación de sustitución es aceptable, factible, asequible, sostenible y segura (AFASS). ${ }^{6}$. Si no se dan estas circunstancias, se aconseja la lactancia materna exclusiva en los primeros seis meses.
2. Afecciones maternas que podrían justificar que se evite la lactancia de forma temporal:

- Enfermedad grave que hace que la madre no pueda cuidar a su bebé, por ejemplo septicemia.

- Herpes simplex tipo 1 (HSV-1): se debe evitar el contacto directo entre las lesiones en el pecho materno y la boca del bebé hasta que toda lesión activa se haya resuelto.

- Medicación materna:

- Los medicamentos psicoterapéuticos sedantes, antiepilépticos, opioides y sus combinaciones pueden causar efectos colaterales tales como mareo y depresión respiratoria, por lo que deben evitarse si existen alternativas más seguras disponibles?

- Es recomendable evitar el uso de iodo radioactivo-131 debido a que están disponibles opciones más seguras; la madre puede reiniciar la lactancia pasados dos meses de haber recibido esta sustancia.

\footnotetext{
a La opción más apropiada de alimentación para el hijo de una madre infectada con el VIH depende de las circunstancias individuales de ella y de su bebé, incluido su estado de salud, pero se deben tener en cuenta los servicios de salud disponibles y los apoyos que pueda recibir. Se recomienda la lactancia materna exclusiva durante los primeros seis meses de vida, a menos que la alimentación de sustitución sea AFASS. Cuando la alimentación de sustitución es AFASS, se debe evitar todo tipo de lactancia materna. La madre infectada con el VIH debe evitar la lactancia mixta (es decir lactancia además de otros alimentos, líquidos o fórmula) durante los primeros seis meses de vida.
} 
- El uso excesivo de yodo o yodóforos tópicos (yodo-povidona), especialmente en heridas abiertas o membranas mucosas, puede provocar supresión tiroidea o anormalidades electrolíticas en el bebé amamantado y deberían evitarse.

- La quimioterapia citotóxica requiere que la madre suspenda el amamantamiento durante la terapia.

3. Afecciones maternas durante las cuales puede continuar la lactancia, aunque representan problemas de salud preocupantes:

- Absceso mamario: el amamantamiento debería continuar con el lado no afectado; el amamantamiento con el pecho afectado puede reiniciarse una vez se ha iniciado el tratamiento ${ }^{8}$.

- Hepatitis B: los lactantes deben recibir la vacuna de la hepatitis B en las primeras 48 horas o apenas sea posible después?.

- Hepatitis C.

- Mastitis: si la lactancia es muy dolorosa, debe extraerse la leche para evitar que progrese la afección ${ }^{8}$.

- Tuberculosis: el manejo de la enfermedad de la madre y el bebé debe hacerse de acuerdo con las normas nacionales ${ }^{10}$.

- Abuso de sustancias, ${ }^{\mathrm{b}, 11}$ : se ha demostrado que el uso materno de nicotina, alcohol, éxtasis, anfetaminas, cocaína y estimulantes relacionados tiene un efecto dañino en los bebés amamantados; el alcohol, los opioides, las benzodiazepinas y el cannabis pueden causar sedación tanto en la madre como en el bebé. Se debe motivar a las madres a no utilizar estas sustancias y darles oportunidades y apoyo para abstenerse.

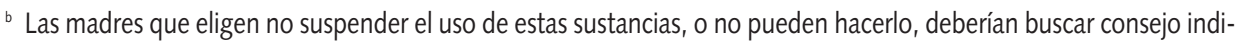
vidual sobre los riesgos y beneficios de la lactancia en función de sus circunstancias individuales. Para las madres que utilizan estas sustancias durante periodos cortos, se debe considerar la suspensión temporal de la lactancia materna durante el tiempo que usen dichas sustancias.
} 


\section{Bibliografía}

1. Technical updates of the guidelines on Integrated Management of Childhood Illness (IMCI). Evidence and recommendations for further adaptations. Ginebra: Organización Mundial de la Salud; 2005.

2. Evidence on the long-term effects of breastfeeding: systematic reviews and meta-analyses. Ginebra, Organización Mundial de la Salud, 2007.

3. León-Cava N, Lutter C, Ross J, Martin L. Cuantificación de los beneficios de la lactancia materna: Reseña de la evidencia. Washington, DC: Organización Panamericana de la Salud; 2002. Disponible en http://www.paho.org/spanish/ad/ fch/BOB-Main.htm (consultado el 04/12/2008).

4. Resolución AMS39.28. Alimentación del Lactante y del Niño Pequeño. En: 33. ${ }^{a}$ Asamblea Mundial de la Salud, Ginebra, 5-16 mayo de 1986. Volumen 1. Resoluciones y documentos. Final. Ginebra: Organización Mundial de la Salud; 1986 (WHA39/1986/REC/1), Anexo 6:122-135.

5. Hypoglycaemia of the newborn: review of the literature. Ginebra: Organización Mundial de la Salud; 1997 (WHO/CHD/97.1). Disponible en http://whqlibdoc.who.int/hq/1997/WHO_CHD_ 97.1.pdf (consultado el 24/06/2008).

6. VIH y alimentación infantil: actualización basada en la reunión consultiva técnica realizada en nombre del Equipo de Trabajo Interinstitucional
(IATT) sobre la Prevención de la Transmisión del VH en Embarazadas, Madres y Niños. Ginebra 2527 de octubre de 2006. Ginebra: Organización Mundial de la Salud; 2008. Disponible en http:// whqlibdoc.who.int/publications/2008/97892 43595962_spa.pdf (Consultado el 04/12/2008).

7. Breastfeeding and maternal medication: recommendations for drugs in the Eleventh WHO Model List of Essential Drugs. Ginebra: Organización Mundial de la Salud; 2003.

8. Mastitis: causas y manejo. Ginebra: Organización Mundial de la Salud; 2000 (WHO/FCH/ $\mathrm{CAH} / 00.13)$. Disponible en http://whqlibdoc. who.int/hq/2000/WHO_FCH_CAH_00.13_spa.p df (consultado el 04/12/2008).

9. Hepatitis B and breastfeeding. Ginebra: Organización Mundial de la Salud; 1996. (Update N. ${ }^{\circ}$ 22).

10. Breastfeeding and Maternal tuberculosis. Ginebra: Organización Mundial de la Salud; 1998 (Update N. ${ }^{\circ} 23$ ).

11. Background papers to the national clinical guidelines for the management of drug use during pregnancy, birth and the early development years of the newborn. Commissioned by the Ministerial Council on Drug Strategy under the Cost Shared Funding Model. North Sydney, Australia: NSW Department of Health; 2006. Disponible en http:/ /www.health.nsw.gov.au/pubs/2006/bkg_preg nancy.html (consultado el 24/06/2008). 